\title{
Influence of beam collimation on fast-atom diffraction studied via a semiquantum approach
}

\author{
M. S. Gravielle* and J. E. Miraglia \\ 4 Instituto de Astronomía y Física del Espacio (IAFE, CONICET-UBA), casilla de correo 67, sucursal 28, C1428EGA, Buenos Aires, Argentina \\ (Received 16 October 2015; published xxxxxx)
}

\begin{abstract}
The influence of the collimating conditions of the incident beam on diffraction patterns produced by grazing scattering of fast atoms off crystal surfaces is studied within a semiquantum approach, called the surface initial value representation (SIVR) approximation. In this approach we incorporate a realistic description of the incident particle in terms of the collimating parameters, which determine the surface area that is coherently illuminated. The model is applied to He atoms colliding with a $\mathrm{LiF}(001)$ surface after passing through a rectangular aperture. As was experimentally observed [Nucl. Instrum. Methods Phys. Res., Sect. B 350, 99 (2015)], SIVR spectra as a function of the azimuthal angle are very sensitive to the width of the collimating slit. We also found that the length of the collimating aperture affects polar angle distributions, introducing additional interference structures for the longer collimating slits.
\end{abstract}

DOI: 10.1103/PhysRevA.00.002700

PACS number(s): $34.35 .+\mathrm{a}, 79.20 . \mathrm{Rf}, 37.25 .+\mathrm{k}$

\section{INTRODUCTION}

Diffraction patterns produced by grazing scattering of swift atoms and molecules (with energies in the $\mathrm{keV}$ range) on surfaces are nowadays becoming a powerful surface analysis tool, which is giving rise to a technique known as grazingincidence fast-atom diffraction (GIFAD or FAD) [1,2]. In recent years the FAD method was successfully applied to very different kinds of materials, ranging from insulators [3-5] to semiconductors [6,7] and metals [8-10], as well as structured films [11] and molecules [12] adsorbed on surfaces. However, in spite of the extensive experimental and theoretical work devoted to the research of FAD since its first experimental observation [3,4], the complete understanding of the underlying quantum processes is far from being achieved. In particular, the study of the mechanisms that contribute to the coherence or decoherence of the scattered particles is still in its infancy.

The observation of quantum interference effects for fast atoms impinging on crystal surfaces strongly relies on the preservation of quantum coherence [13-15] and, in this regard, the coherence conditions of the incident beam play an important role. Motivated by Ref. [16], in this article we investigate the influence of the collimation of the incident beam on FAD patterns by making use of a recently developed approach called the surface initial value representation (SIVR) approximation [17]. With this goal we explicitly take into account the experimental collimating conditions to determine the surface region that is coherently illuminated by the particle beam and use this information to build the initial wave packet that describes the unperturbed state of the incident particle within the SIVR method.

The SIVR approximation is a semiquantum approach that was derived from the initial value representation (IVR) method by Miller [18] by using the corresponding semiquantum time evolution operator in the frame of a time-dependent distortedwave formalism. This strategy incorporates an approximate description of classically forbidden transitions on the dark side of rainbow angles, making it possible to avoid the classical

\footnotetext{
*Author to whom correspondence should be addressed: msilvia@iafe.uba.ar
}

rainbow divergence present in previous semiclassical models 53 for FAD, like the surface-eikonal (SE) approach $[19,20]$. Such 54 a weakness of the SE method affects the intensity of the 55 outermost diffraction maxima when these maxima are close to 56 the classical rainbow angles [10], i.e., the extreme deflection ${ }_{57}$ angles of the classical projectile distribution. The SIVR ${ }_{58}$ approach, instead, provides an appropriate description of FAD 59 patterns along the whole angular range, even around classical 60 rainbow angles, without requiring the use of convolutions 61 to smooth the theoretical curves [17]. Therefore, the SIVR 62 method can be considered as an attractive alternative to quan- 63 tum wavepacket propagations, offering a clear representation 64 of the main mechanisms of the process in terms of classical 65 trajectories through the Feynman path integral formulation of 66 quantum mechanics.

In order to analyze the influence of the beam collima- 68 tion on FAD spectra, an extended version of the SIVR 69 approximation -including the collimating parameters-is ap- 70 plied to evaluate FAD patterns for He atoms grazingly imping- 71 ing on a $\operatorname{LiF}(001)$ surface after going through a rectangular 72 aperture. The paper is organized as follows: The theoretical 73 formalism is summarized in Sec. II. Results for different sizes 74 of the collimating aperture are presented and discussed in 75 Sec. III, while in Sec. IV we outline our conclusions. Atomic 76 units (a.u.) are used unless otherwise stated.

\section{THEORETICAL MODEL}

78

Let us consider an atomic projectile $P$ with initial momen- 79 tum $\vec{K}_{i}$, which is elastically scattered from a crystal surface 80 $S$, ending in a final state with momentum $\vec{K}_{f}$ and total energy 81 $E=K_{f}^{2} /\left(2 m_{P}\right)=K_{i}^{2} /\left(2 m_{P}\right)$, with $m_{P}$ being the projectile 82 mass. By employing the IVR method [21], the scattering state 83 of the projectile at time $t$ can be approximated as [17]

$$
\begin{aligned}
\left|\Psi_{i}^{(\mathrm{SIVR})+}(t)\right\rangle= & \frac{1}{(2 \pi i)^{3 / 2}} \int d \vec{R}_{o} f_{i}\left(\vec{R}_{o}\right) \int d \vec{K}_{o} g_{i}\left(\vec{K}_{o}\right) \\
& \times\left[J_{M}(t)\right]^{1 / 2} \Phi_{i}\left(\vec{R}_{o}\right) \exp \left(i S_{t}\right)\left|\overrightarrow{\mathcal{R}}_{t}\right\rangle
\end{aligned}
$$

where 
86 is the initial momentum eigenfunction, with $\vec{R}$ being the 87 position of the center of mass of the incident atom, and the 88 sign " + " in the supra-index of the scattering state indicates 89 that it satisfies outgoing asymptotic conditions. In Eq. (1) the 90 position ket $\left|\overrightarrow{\mathcal{R}}_{t}\right\rangle$ is associated with the time-evolved position 91 of the incident atom at a given time $t, \overrightarrow{\mathcal{R}}_{t} \equiv \overrightarrow{\mathcal{R}}_{t}\left(\vec{R}_{o}, \vec{K}_{o}\right)$, 92 which is derived by considering a classical trajectory with 93 starting position and momentum $\vec{R}_{o}$ and $\vec{K}_{o}$, respectively. ${ }_{94}$ The function $S_{t}$ denotes the classical action along the trajectory

$$
S_{t}=S_{t}\left(\vec{R}_{o}, \vec{K}_{o}\right)=\int_{0}^{t} d t^{\prime}\left[\frac{\overrightarrow{\mathcal{P}}_{t^{\prime}}^{2}}{2 m_{P}}-V_{\mathrm{SP}}\left(\overrightarrow{\mathcal{R}}_{t^{\prime}}\right)\right] \text {, }
$$

95 with $\overrightarrow{\mathcal{P}}_{t}=m_{P} d \overrightarrow{\mathcal{R}}_{t} / d t$ being the classical projectile mo96 mentum at the time $t$ and $V_{\mathrm{SP}}$ being the surface-projectile 97 interaction, while the function

$$
J_{M}(t)=\operatorname{det}\left[\frac{\partial \overrightarrow{\mathcal{R}}_{t}\left(\vec{R}_{o}, \vec{K}_{o}\right)}{\partial \vec{K}_{o}}\right]
$$

98 is a Jacobian factor (a determinant) evaluated along the 99 classical trajectory $\overrightarrow{\mathcal{R}}_{t}$. This Jacobian factor can be related 100 to the Maslov index [22] by expressing it as $J_{M}(t)=$ $101\left|J_{M}(t)\right| \exp \left(i v_{t} \pi\right)$, where $\left|J_{M}(t)\right|$ is the modulus of $J_{M}(t)$ and $102 v_{t}$ is an integer number that accounts for the sign of $J_{M}(t)$ 103 at a given time $t$. In this way, $v_{t}$ represents a time-dependent 104 Maslov index, satisfying the condition that, every time that $105 J_{M}(t)$ changes its sign along the trajectory, $v_{t}$ increases by 1.

106 The functions $f_{i}\left(\vec{R}_{o}\right)$ and $g_{i}\left(\vec{K}_{o}\right)$, present in the integrand 107 of Eq. (1), describe the shape of the position and momentum 108 wave packet associated with the incident projectile. In a 109 previous paper [17] $f_{i}\left(\vec{R}_{o}\right)$ was considered as a Gaussian 110 distribution illuminating a fixed number of reduced unit 111 cells of the crystal surface, while $g_{i}\left(\vec{K}_{o}\right)$ was defined as a 112 uniform distribution. Here these functions are derived from 113 the collimation conditions of the incident beam in order to 114 incorporate a realistic profile of the coherent initial wave 115 packet, as explained in the following section.

${ }_{116}$ By using the SIVR scattering state, given by Eq. (1), 117 within the framework of the time-dependent distorted-wave 118 formalism [23], the SIVR transition amplitude, per unit of 119 surface area $\mathcal{S}$, can be expressed as [17]

$$
\begin{aligned}
A_{i f}^{(\mathrm{SIVR})}= & \frac{1}{\mathcal{S}} \int_{\mathcal{S}} d \vec{R}_{o} f_{i}\left(\vec{R}_{o}\right) \int d \vec{K}_{o} g_{i}\left(\vec{K}_{o}\right) \\
& \times a_{i f}^{(\mathrm{SIVR})}\left(\vec{R}_{o}, \vec{K}_{o}\right),
\end{aligned}
$$

120 where

$$
\begin{aligned}
a_{i f}^{(\mathrm{SIVR})}\left(\vec{R}_{o}, \vec{K}_{o}\right)= & -\int_{0}^{+\infty} d t \frac{\left|J_{M}(t)\right|^{1 / 2} e^{i v_{t} \pi / 2}}{(2 \pi i)^{9 / 2}} V_{\mathrm{SP}}\left(\overrightarrow{\mathcal{R}}_{t}\right) \\
& \times \exp \left[i\left(\varphi_{t}^{(\mathrm{SIVR})}-\vec{Q} \cdot \vec{R}_{o}\right)\right]
\end{aligned}
$$

121 is the partial transition amplitude associated with the classi122 cal path $\overrightarrow{\mathcal{R}}_{t} \equiv \overrightarrow{\mathcal{R}}_{t}\left(\vec{R}_{o}, \vec{K}_{o}\right)$, with $\vec{Q}=\vec{K}_{f}-\vec{K}_{i}$ being the 123 projectile momentum transfer and

$$
\varphi_{t}^{(\mathrm{SIVR})}=\int_{0}^{t} d t^{\prime}\left[\frac{1}{2 m_{P}}\left(\vec{K}_{f}-\overrightarrow{\mathcal{P}}_{t^{\prime}}\right)^{2}-V_{\mathrm{SP}}\left(\overrightarrow{\mathcal{R}}_{t^{\prime}}\right)\right]
$$

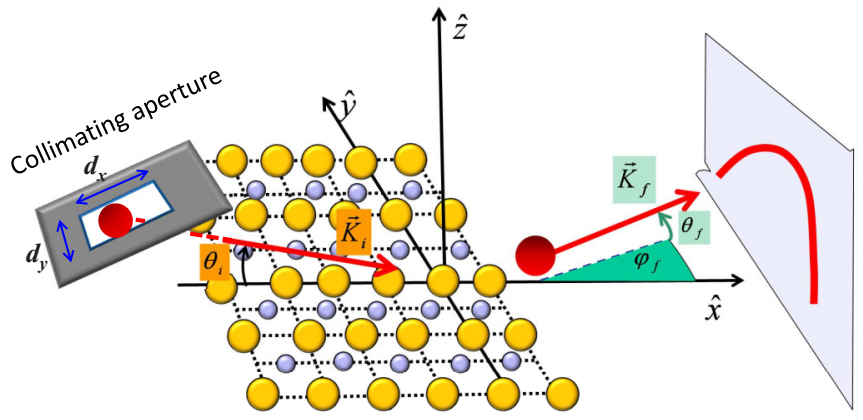

(a)

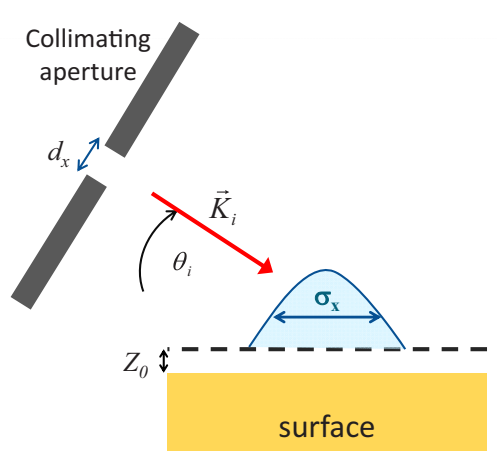

FIG. 1. (Color online) (a) Sketch of the FAD process, including the collimating aperture. (b) Lateral sight of the scattering process.

being the SIVR phase at the time $t$. Details of the derivation ${ }_{124}$ of the SIVR method are given in Ref. [17].

In this article we use a frame of reference placed on the ${ }_{126}$ first atomic layer, with the surface contained in the $x-y$ plane, 127 the $\widehat{x}$ versor along the incidence direction and the $\hat{z}$ versor ${ }_{128}$ oriented perpendicular to the surface, aiming towards the 129 vacuum region. The SIVR differential probability, per unit ${ }_{130}$ of surface area, for elastic scattering with final momentum $\vec{K}_{f}{ }_{131}$ in the direction of the solid angle $\Omega_{f} \equiv\left(\theta_{f}, \varphi_{f}\right)$, is obtained ${ }_{132}$ from Eq. (5) as

$$
d P^{(\mathrm{SIVR})} / d \Omega_{f}=K_{f}^{2}\left|A_{i f}^{(\mathrm{SIVR})}\right|^{2}
$$

where $\theta_{f}$ and $\varphi_{f}$ are the final polar and azimuthal angles, 134 respectively, with $\theta_{f}$ measured with respect to the surface and 135 $\varphi_{f}$ measured with respect to the $\widehat{x}$ axis. A schematic depiction 136 of the process and the coordinates is displayed in Fig. 1(a). ${ }_{137}$

\section{Size of initial coherent wave packet}

In Eq. (5), the variables $\vec{R}_{o}$ and $\vec{K}_{o}$ represent the starting 139 position and momentum, respectively, of the classical projec- 140 tile trajectory, both measured at $t=0$, while the functions 141 $f_{i}\left(\vec{R}_{o}\right)$ and $g_{i}\left(\vec{K}_{o}\right)$ determine the shape of the initial wave 142 packet, satisfying the Heisenberg uncertainty relation. We ${ }_{143}$ decompose the starting position as $\vec{R}_{o}=\vec{R}_{o s}+Z_{o} \widehat{z}$, where ${ }_{144}$ $\vec{R}_{o s}=X_{o} \widehat{x}+Y_{o} \widehat{y}$ and $Z_{o}$ are the components parallel and ${ }_{145}$ perpendicular, respectively, to the surface plane, with $Z_{o}$ being 146 a fixed distance for which the projectile is hardly affected by 147 the surface interaction. 132 3

\section{6} (n) . . (n) 
149 We assume that the size of the coherent initial wave 150 packet, at a distance $Z_{o}$ from the surface, is governed by 151 the collimation of the incident beam as given by the Van 152 Cittert-Zernike theorem [24]. By considering a rectangular 153 collimating aperture placed a long distance $L$ from the surface, 154 the coherence size of the incident beam on the $Z_{o}$ plane, which 155 is located parallel to the surface at a distance $Z_{o}$ from it, 156 is defined by the complex grade of coherence, $\mu\left(X_{o}, Y_{o}\right)$. It 157 reads [24]

$$
\left|\mu\left(X_{o}, Y_{o}\right)\right|^{2}=j_{0}^{2}\left(\frac{\pi d_{x}}{\lambda_{\perp} L^{\prime}} X_{o}\right) j_{0}^{2}\left(\frac{\pi d_{y}}{\lambda L^{\prime}} Y_{o}\right),
$$

158 where $j_{0}(x)$ is the spherical Bessel function and $d_{x}$ and $d_{y}$ 159 denote the lengths of the sides of the rectangular aperture, 160 which form angles $\theta_{x}=\pi / 2-\theta_{i}$ and $\theta_{y}=0$, respectively, 161 with the surface plane, and $\theta_{i}$ being the glancing incidence 162 angle [see Figs. 1(a) and 1(b)]. In Eq. (9) the de Broglie 163 wavelengths $\lambda$ and $\lambda_{\perp}$ are defined as

$$
\lambda=2 \pi / K_{i} \text { and } \quad \lambda_{\perp}=\lambda / \sin \theta_{i},
$$

164 respectively, this last one being associated with the initial 165 motion normal to the surface plane, while $L^{\prime}=L-Z_{o} / \sin \theta_{i}$. 166 For most of the collision systems, $Z_{o}$ can be chosen as equal 167 to the lattice constant of the crystal, leading to $L^{\prime} \cong L$.

According Eq. (9) the spatial profile of the initial wave 169 packet can be approximated by a product of Gaussian 170 functions,

$$
G(\omega, x)=\left[2 /\left(\pi \omega^{2}\right)\right]^{1 / 4} \exp \left(-x^{2} / \omega^{2}\right),
$$

171 as follows:

$$
f_{i}\left(\vec{R}_{o s}\right)=G\left(\sigma_{x}, X_{o}\right) G\left(\sigma_{y}, Y_{o}\right),
$$

172 where the parameters $\sigma_{x}$ and $\sigma_{y}$ were derived by fitting the 173 complex grade of coherence, i.e., $\left|\mu\left(X_{o}, Y_{o}\right)\right|^{2} \simeq\left|f_{i}\left(\vec{R}_{o s}\right)\right|^{2}$, 174 reading

$$
\sigma_{x}=\frac{\lambda \perp}{\sqrt{2}} \frac{L}{d_{x}}, \quad \sigma_{y}=\frac{\lambda}{\sqrt{2}} \frac{L}{d_{y}} .
$$

175 The lengths $\sigma_{x}$ and $\sigma_{y}$ represent the effective widths of 176 the $\left|G\left(\sigma_{x}, X_{o}\right)\right|^{2}$ and $\left|G\left(\sigma_{y}, Y_{o}\right)\right|^{2}$ distributions, respectively, 177 being defined as the corresponding root-mean-square devi178 ations [25]. Notice that these widths are associated with 179 the transversal coherence size of the initial wave packet, 180 a magnitude that is crucial in matter-wave interferometry 181 [26-28].

182 On the other hand, concerning the momentum profile of 183 the initial wave packet, as we are dealing with an incident 184 beam with a well-defined energy, i.e., $\Delta E / E \ll 1[16]$, the 185 longitudinal coherence length does not play any role [26]. ${ }_{186}$ Consequently, the starting momentum $\vec{K}_{o}$ satisfies energy 187 conservation, with $K_{0}=\left|\vec{K}_{0}\right|=\sqrt{2 m_{P} E}$, and the integration 188 on $\vec{K}_{0}$ can be solved by making use of the change of variables ${ }_{189} \vec{K}_{o}=K_{o}\left(\cos \theta_{o} \cos \varphi_{o}, \cos \theta_{o} \sin \varphi_{o},-\sin \theta_{o}\right)$, with $\theta_{o}$ and $190 \varphi_{o}$ varying around the incidence angles $\theta_{i}$ and $\varphi_{i}=0$, 191 respectively. The shape of the corresponding angular wave 192 packet is described again in terms of Gaussian functions, 193 reading

$$
g_{i}\left(\vec{K}_{o}\right) \simeq g_{i}\left(\Omega_{o}\right)=G\left(\sigma_{\theta}, \theta_{o}-\theta_{i}\right) G\left(\sigma_{\varphi}, \varphi_{o}\right),
$$

where $\Omega_{o} \equiv\left(\theta_{o}, \varphi_{o}\right)$ is the solid angle corresponding to the $\vec{K}_{o} 194$ direction and the angular widths of the $\theta_{o}$ and $\varphi_{o}$ distributions 195 were derived from the uncertainty principle as [25]

$$
\sigma_{\theta}=\frac{\lambda_{\perp}}{2 \sigma_{x}} \quad \text { and } \quad \sigma_{\varphi}=\frac{\lambda}{2 \sigma_{y}}
$$

respectively.

Replacing Eqs. (12) and (14) in Eq. (5), the extended 198 version of the SIVR transition amplitude, including explicitly ${ }_{199}$ the proper shape of the incident wave packet, is expressed as 200

$$
A_{i f}^{(\mathrm{SIVR})}=\frac{\alpha}{\mathcal{S}} \int_{\mathcal{S}} d \vec{R}_{o s} f_{i}\left(\vec{R}_{o s}\right) \int d \Omega_{o} g_{i}\left(\Omega_{o}\right) a_{i f}^{(\mathrm{SIVR})}\left(\vec{R}_{o}, \vec{K}_{o}\right) \text {, }
$$

where $a_{i f}^{(\mathrm{SIVR})}\left(\vec{R}_{o}, \vec{K}_{o}\right)$ is given by Eq. (6) and $\alpha=m_{P} K_{i}$.

\section{RESULTS}

We apply the extended SIVR method to ${ }^{4} \mathrm{He}$ atoms 203 elastically scattered from a $\mathrm{LiF}(001)$ surface under axial 204 surface channeling conditions since, for this collision system, 205 diffraction patterns for different widths of the collimating slit 206 were reported in Ref. [16]. The SIVR transition amplitude 207 was obtained from Eq. (16) by employing the Monte Carlo 208 technique to evaluate the $\vec{R}_{o s}$ and $\Omega_{o}$ integrals, considering 209 more than $4 \times 10^{5}$ points in such an integration. For every 210 starting point, the partial transition amplitude $a_{i f}^{(\text {SIVR) }}\left(\vec{R}_{o}, \vec{K}_{o}\right){ }_{211}$ was evaluated numerically from Eq. (6) by employing a 212 potential $V_{\mathrm{SP}}$ derived from a pairwise additive hypothesis. 213 The potential model used in this work is the same as the 214 one employed in Ref. [17]. It describes the surface-projectile 215 interaction as the sum of the static and polarization contribu- 216 tions, the first of them evaluated incorporating no local terms 217 of the electronic density in the kinetic and exchange potentials. 218 The potential $V_{\text {SP }}$ also takes into account a surface rumpling, 219 with a displacement distance extracted from Ref. [20]. Details 220 of the surface potential will be published elsewhere [29]. 221

In this work we vary the size of the collimating aperture, 222 keeping a fixed incidence condition given by helium projectiles 223 impinging along the $\langle 110\rangle$ channel with a total energy $E={ }_{224}$ $1 \mathrm{keV}$ and an incidence angle $\theta_{i}=0.99^{\circ}$. In all the cases, 225 the distance between the collimating aperture and the surface 226 is chosen as $L=25 \mathrm{~cm}$, in agreement with the experimental 227 setup of Ref. [16].

In Figs. 2 and 3 we show two-dimensional projectile 229 distributions, as a function of $\theta_{f}$ and $\varphi_{f}$, derived within 230 the SIVR approximation by considering collimation slits 231 with the same length- $d_{x}=1.5 \mathrm{~mm}$-but two different ${ }_{232}$ widths: $d_{y}=0.2 \mathrm{~mm}$ and $d_{y}=1.0 \mathrm{~mm}$, respectively. Both 233 SIVR distributions reproduce quite well the corresponding 234 experimental distributions [16], which are also displayed in the 235 figures. They present the usual banana shape, characteristic of 236 the axial surface scattering [30], with final dispersion angles 237 lying on a thick annulus, whose mean radius is approximately 238 equal to $\theta_{i}$. From the comparison of Figs. 2 and 3 it is 239 clearly observed that the width of the collimation slit strongly 240 affects the diffraction patterns, making the well-defined peaks 241 present in the distributions of Fig. 2, for the more narrow slit, 242 completely disappear when the width of the slit is increased, 243 


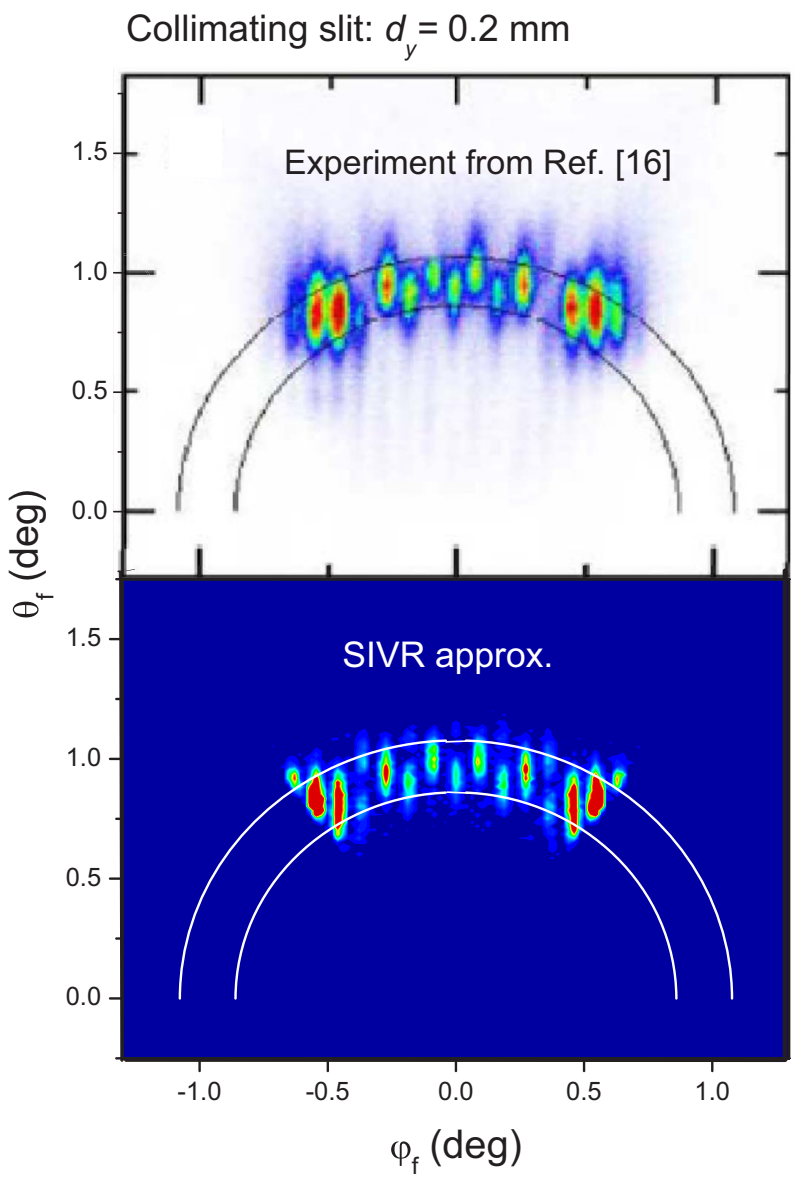

FIG. 2. (Color online) Two-dimensional projectile distribution as a function of the final dispersion angles $\theta_{f}$ and $\varphi_{f}$, for $1 \mathrm{keV}$ ${ }^{4} \mathrm{He}$ atoms impinging on $\operatorname{LiF}(001)$ along the $\langle 110\rangle$ direction with the incidence angle $\theta_{i}=0.99^{\circ}$. The incident helium beam is collimated with a rectangular aperture of sides $d_{x}=1.5 \mathrm{~mm}$ and $d_{y}=$ $0.2 \mathrm{~mm}$. Upper panel shows experimental distribution extracted from Ref. [16]; lower panel shows SIVR distribution.

244 as happens in Fig. 3. In the experimental and theoretical 245 intensity distributions of Fig. 3, only the maxima at the rainbow 246 deflection angles $\pm \Theta_{r b}$ are visible. As discussed in Ref. [16], 247 this behavior is related to the area $\mathcal{S}$ of the surface plane that 248 is coherently lighted by the incident beam and will be studied 249 in detail within the SIVR approach.

$250 \quad$ In Eq. (16), by splitting the $\vec{R}_{o s}$ integral over the area $\mathcal{S}$ 251 into a collection of integrals over different reduced unit cells, it 252 is possible to express $A_{i f}^{\text {(SIVR) }}$ as a product of two factors [17]:

$$
A_{i f}^{(\mathrm{SIVR})} \simeq A_{i f, 1}^{(\mathrm{SIVR})} F_{B}
$$

253 each of them associated with a different interference mech254 anism. The factor $A_{i f, 1}^{\text {(SIVR) }}$, called a unit-cell form factor, is 255 derived from Eq. (16) by evaluating the $\vec{R}_{o s}$ integral over 256 only one reduced unit cell, being related to supernumerary 257 rainbows [31]. While the factor $F_{B}$ is a crystallographic factor 258 associated with Bragg diffraction, which originates from the 259 interference of identical trajectories whose initial positions ${ }_{260} \vec{R}_{o s}$ are separated by a distance equal to the spatial periodicity
Collimating slit: $d_{y}=1.0 \mathrm{~mm}$

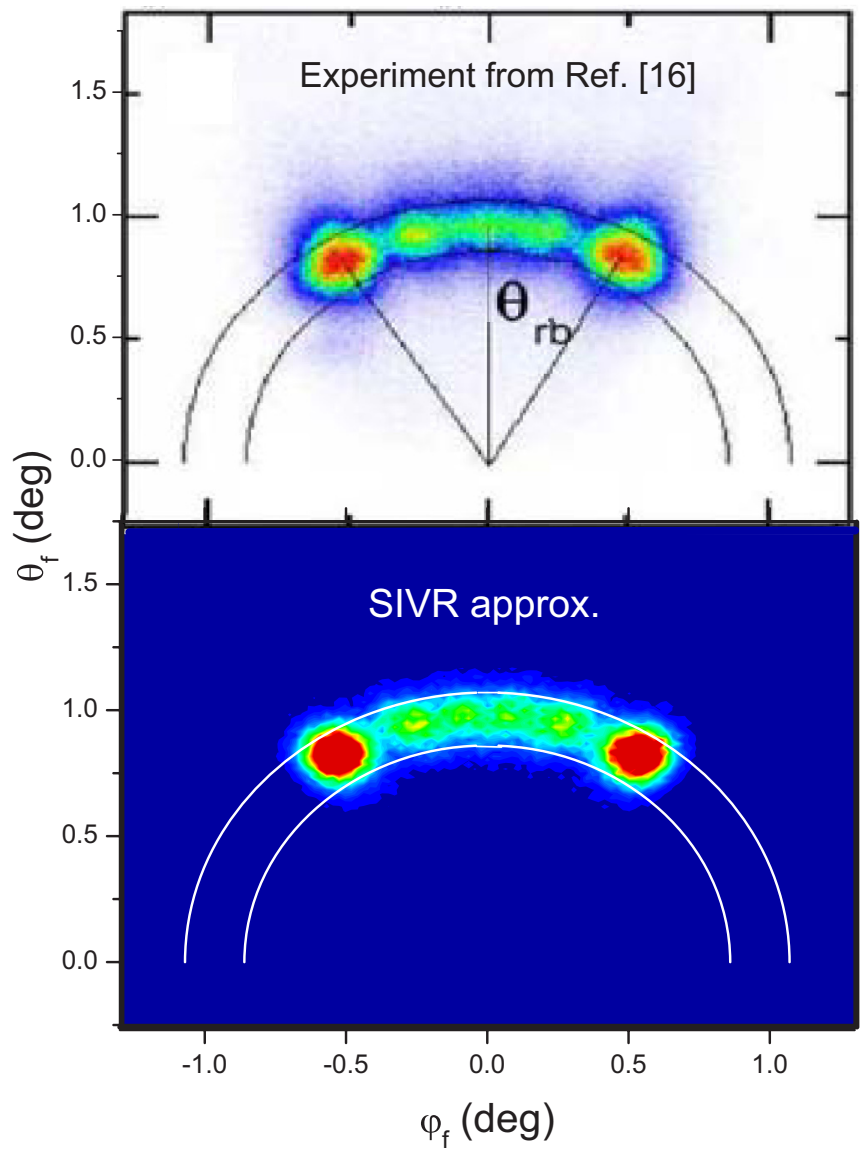

FIG. 3. (Color online) Similar to Fig. 2 for a collimating aperture of sides $d_{x}=1.5 \mathrm{~mm}$ and $d_{y}=1.0 \mathrm{~mm}$. The radial lines in the upper panel indicate the positions of the rainbow deflection angles $\pm \Theta_{r b}$.

of the lattice. The factor $F_{B}$ depends on $\vec{Q}$ and the area $\mathcal{S}{ }_{261}$ coherently illuminated by the particle beam, being insensible 262 to the potential model.

In Eq. (16) the effective area $\mathcal{S}$ coherently lighted by 264 the incident beam can be estimated as $\mathcal{S} \simeq \mathcal{D}_{x} \mathcal{D}_{y}$, where ${ }_{265}$ the distances $\mathcal{D}_{j}=2 \sqrt{2} \sigma_{j}$ with $j=x, y$ were determined ${ }_{26}$ from the $\left(X_{o}, Y_{o}\right)$ values for which the function $\left|\mu\left(X_{o}, Y_{o}\right)\right|^{2}, \quad 267$ given by Eq. (9), vanishes. Under typical incidence conditions 268 for FAD, the dependence of $F_{B}$ on the azimuthal angle $\varphi_{f}{ }_{269}$ becomes completely governed by the number $n_{y}$ of reduced 270 unit cells in the direction transversal to the incidence channel 271 that are coherently illuminated by the initial wave packet, i.e., 272 $n_{y} \simeq \mathcal{D}_{y} / a_{y}$, where $a_{y}$ is the length of the reduced unit cell 273 along the $\hat{y}$ direction. For $n_{y} \gtrsim 2$ the factor $F_{B}$ gives rise 274 to Bragg peaks placed at azimuthal angles that verify the 275 relation $\sin \varphi_{f}=m \lambda / a_{y}$, with $m$ being an integer, as observed 276 in Fig. 2 where $n_{y} \simeq 4$. The relative intensities of theses Bragg 277 peaks are modulated by $A_{i f, 1}^{\text {(SIVR) }}$, which acts as an envelope 278 function that can reduce or even suppress the contribution 279 of a given Bragg order, while the peak width is determined 280 by $n_{y}$, narrowing as $n_{y}$ increases. But when the coherently 281 illuminated region shrinks to cover around a reduced unit cell 282 in the transversal direction, only the unit-cell factor is present 283 


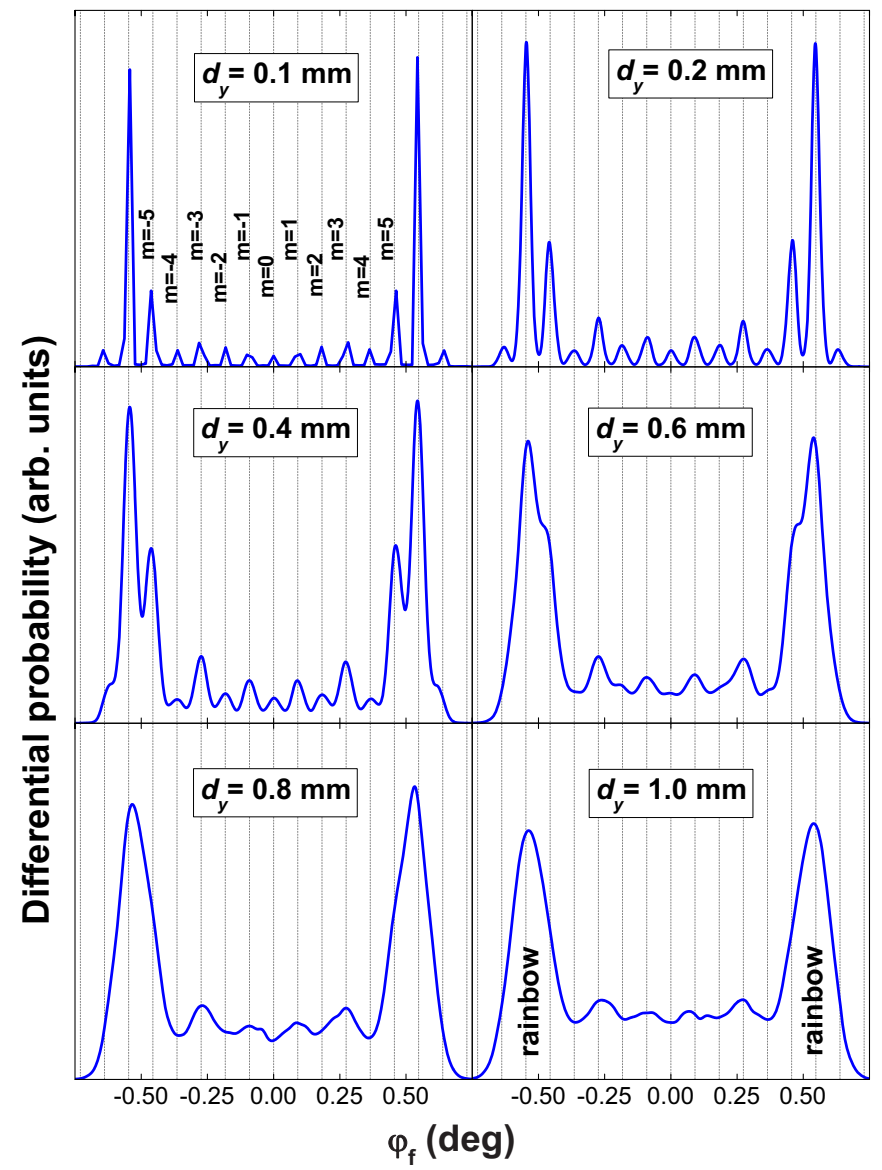

FIG. 4. (Color online) Azimuthal angular distribution as a function of $\varphi_{f}$ for $1 \mathrm{keV}{ }^{4} \mathrm{He}$ atoms impinging on $\mathrm{LiF}(001)$ along the $\langle 110\rangle$ direction with the incidence angle $\theta_{i}=0.99^{\circ}$. The incident helium beam is collimated with a rectangular aperture of length $d_{x}=$ $1.5 \mathrm{~mm}$ and different widths: $d_{y}=0.1,0.2,0.4,0.6,0.8$, and $1.0 \mathrm{~mm}$, respectively. Vertical lines indicate the angular positions of Bragg peaks, as explained in the text.

284 in Eq. (17). Consequently, the angular distribution shows 285 structures associated with supernumerary rainbow maxima 286 exclusively, as it happens in Fig. 3 where $n_{y} \lesssim 1$. 288 the diffraction patterns with the width of the slit, in Fig. 4 289 we display the differential probability $d P^{(\mathrm{SIVR})} / d \varphi_{f}$, as a 290 function of the azimuthal angle $\varphi_{f}$, for different values of $291 d_{y}$. As given by Eq. (13), when $d_{y}$ augments, the number $292 n_{y}$ of the coherently illuminated cells decreases while the 293 width of the Bragg peaks increases, as observed in Fig. 4 294 for $d_{y} \lesssim 0.4 \mathrm{~mm}$. For wider collimating slits Bragg peaks start 295 to blur out, disappearing completely for $d_{y}=0.8 \mathrm{~mm}$, where $296 n_{y} \simeq 1$. Therefore, varying $d_{y}$ we can inspect two different 297 zoologies: Bragg peaks at small $d_{y}$ values and supernumerary 298 rainbow peaks at large $d_{y}$.

We also investigate the influence of the length of the collimating aperture, $d_{x}$, on FAD patterns. In Fig. 5 we display angular projectile distributions derived from the SIVR approach by considering a collimating slit with the same width, $d_{y}=0.2 \mathrm{~mm}$, and three different lengths: $d_{x}=0.2,2.0$, and $3044.0 \mathrm{~mm}$. For a small square aperture [Fig. 5(a)], Bragg peaks

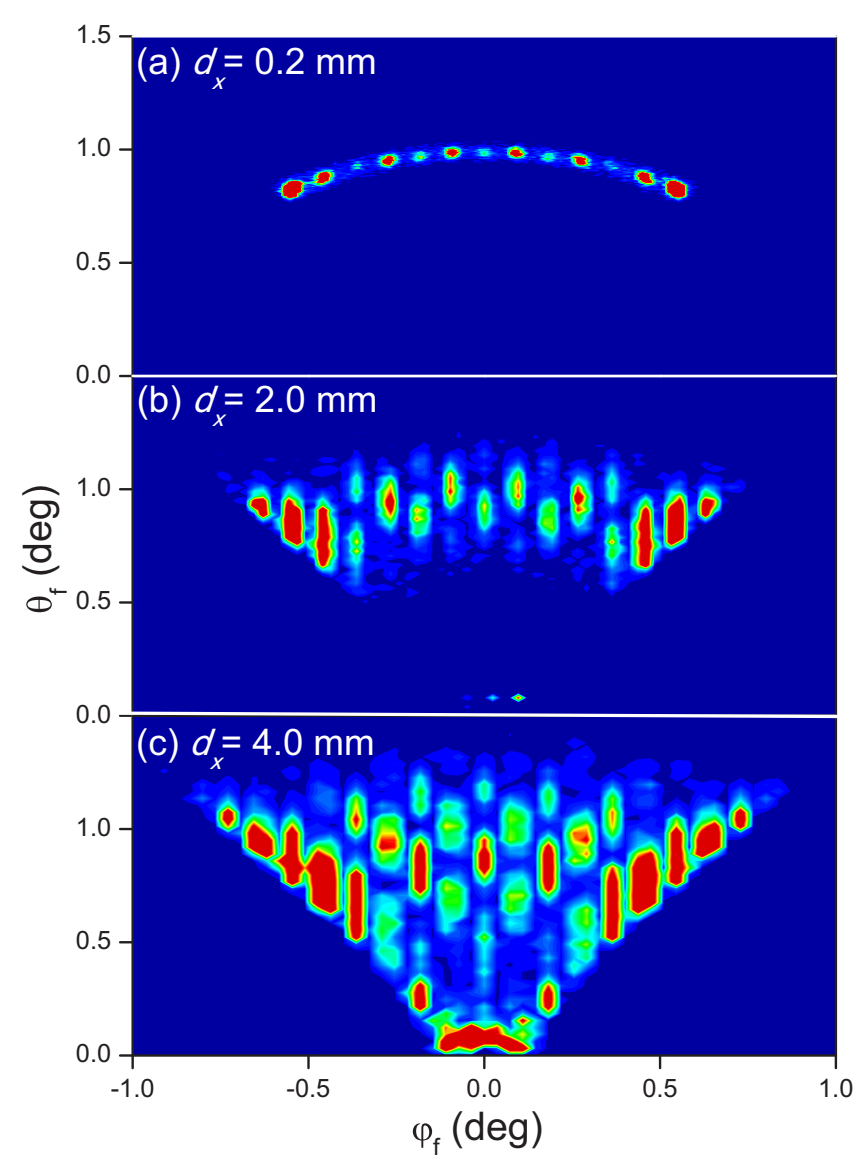

FIG. 5. (Color online) Similar to Fig. 2 for a collimating slit of width $d_{y}=0.2 \mathrm{~mm}$ and different lengths: (a) $d_{x}=0.2 \mathrm{~mm}$, (b) $d_{x}=$ $2.0 \mathrm{~mm}$, and (c) $d_{x}=4.0 \mathrm{~mm}$.

are observed like circular spots lying on a thin ring whose 305 radius is equal to $\theta_{i}$, corresponding to an almost ideal elastic 306 rebound $\vec{K}_{i} \rightarrow \vec{K}_{f}$. But when the length of the collimating 307 aperture augments up to $d_{x}=2.0 \mathrm{~mm}$ [Fig. 5(b)], transforming 308 the square orifice into a slit, Bragg peaks become visible ${ }_{309}$ like elongated strips which are placed at slightly different 310 radius. This effect is even more evident in Fig. 5(c) for 311 $d_{x}=4.0 \mathrm{~mm}$, where the projectile distribution resembles the 312 diffraction charts for different normal energies $E_{\perp}=E \sin ^{2} \theta_{i}$. ${ }_{313}$ The explanation is simple: from Eqs. (13) and (15), if $d_{x}$ is ${ }_{314}$ large $\sigma_{\theta}$ is also large, enabling a wide spread of the impact 315 momentum normal to the surface plane, $\left|K_{o z}\right|=K_{o} \sin \theta_{o} . \quad 316$ Such a $K_{o z}$ dispersion gives rise to the structures along the ${ }_{317}$ vertical axis of Fig. 5(c). Hence, the intensity oscillations along 318 the $\theta_{f}$ axis observed for long collimating slits are probing the ${ }_{319}$ surface potential for different distances to the topmost atomic 320 plane. They might be a useful tool to explore different distances 321 to the surface without varying the mean value of the normal 322 energy $E_{\perp}$.

The previous analysis was done by keeping the de Broglie 324 wavelengths of Eq. (10) constant. However, the size of the 325 coherently illuminated region is affected by the $\lambda$ and $\lambda_{\perp}{ }_{326}$ values, as given by Eq. (13). Then, in FAD experiments, the 327 dimensions of the collimating aperture should be modified for 328 every incidence condition in order to ensure a similar coherent ${ }_{329}$ 
330 lighting of the surface in all cases. Additionally, notice that the 331 transversal coherence length $\sigma_{x}\left(\sigma_{y}\right)$ depends on the ratio $L / d_{x}$ ${ }_{332}\left(L / d_{y}\right)$, so that any change of the collimating conditions that ${ }_{33}$ keeps this ratio constant would produce the same interference 334 patterns. Furthermore, even though the present results were 335 obtained by considering rectangular collimating apertures, the 336 main outcomes of the work are expected to hold also for 337 circular collimating apertures.

344 surface considering a rectangular collimating aperture with 345 different sizes. As was found experimentally [16], the SIVR 346 interference patterns are strongly affected by the width of the 347 collimating slit, which determines the transversal length of 348 the surface area that is coherently illuminated by the incident 349 wake packet. The number of lighted reduced unit cells in the 350 direction transverse to the incidence channel determines the azimuthal width of the Bragg peaks, making either Bragg peaks 351 or supernumerary rainbows visible. Therefore, knowledge 352 of the experimental collimating conditions is essential for a ${ }_{353}$ meaningful comparison with theoretical distributions.

On the other hand, the length of the collimating slit 355 affects the polar $\theta_{f}$ distribution of scattered projectiles, this 356 effect being related to the dispersion of the component ${ }^{357}$ of the initial momentum perpendicular to the surface. As ${ }_{358}$ the length of the collimating aperture increases, diffraction 359 maxima are transformed from circular spots into elongated 360 strips, where interference structures along the $\theta_{f}$ axis arise ${ }_{361}$ for the longer slits. These findings suggest that collimating 362 slits with several millimeters of length might be used to ${ }_{363}$ probe the projectile-surface interaction for different normal 364 distances. Alternatively, if the usual diffraction charts are 365 employed for surface analysis, sufficiently short collimating 366 apertures are required to ensure a small dispersion of the initial ${ }_{367}$ perpendicular energy.

\section{ACKNOWLEDGMENTS}

The authors acknowledge financial support from CON- 370 ICET, UBA, and ANPCyT of Argentina.

\section{1}

[1] H. Winter and A. Schüller, Prog. Surf. Sci. 86, 169 (2011), and references therein.

[2] A. Zugarramurdi et al., Appl. Phys. Lett. 106, 101902 (2015).

[3] A. Schüller, S. Wethekam, and H. Winter, Phys. Rev. Lett. 98, 016103 (2007).

[4] P. Rousseau, H. Khemliche, A. G. Borisov, and P. Roncin, Phys. Rev. Lett. 98, 016104 (2007).

[5] A. Schüller et al., Surf. Sci. 606, 161 (2012).

[6] H. Khemliche, P. Rousseau, P. Roncin, V. H. Etgens, and F. Finocchi, Appl. Phys. Lett. 95, 151901 (2009).

[7] M. Debiossac, A. Zugarramurdi, H. Khemliche, P. Roncin, A. G. Borisov, A. Momeni, P. Atkinson, M. Eddrief, F. Finocchi, and V. H. Etgens, Phys. Rev. B 90, 155308 (2014).

[8] N. Bundaleski, H. Khemliche, P. Soulisse, and P. Roncin, Phys. Rev. Lett. 101, 177601 (2008).

[9] M. Busch, A. Schüller, S. Wethekam, and H. Winter, Surf. Sci. 603, L23 (2009).

[10] C. A. Ríos Rubiano, G. A. Bocan, M. S. Gravielle, N. Bundaleski, H. Khemliche, and P. Roncin, Phys. Rev. A 87, 012903 (2013).

[11] J. Seifert, A. Schüller, H. Winter, R. Wlodarczyk, J. Sauer, and M. Sierka, Phys. Rev. B 82, 035436 (2010).

[12] J. Seifert, M. Busch, E. Meyer, and H. Winter, Phys. Rev. Lett. 111, 137601 (2013).

[13] F. Aigner, N. Simonović, B. Solleder, L. Wirtz, and J. Burgdörfer, Phys. Rev. Lett. 101, 253201 (2008).

[14] J. Lienemann et al., Phys. Rev. Lett. 106, 067602 (2011).

[15] N. Bundaleski, P. Soulisse, A. Momeni, H. Khemliche, and P. Roncin, Nucl. Instrum. Methods Phys. Res., Sect. B 269, 1216 (2011).
[16] J. Seifert, J. Lienemann, A. Schüller, and H. Winter, Nucl. Instrum. Methods Phys. Res., Sect. B 350, 99 (2015).

[17] M. S. Gravielle and J. E. Miraglia, Phys. Rev. A 90, 052718 (2014).

[18] W. H. Miller, J. Chem. Phys. 53, 3578 (1970).

[19] M. S. Gravielle and J. E. Miraglia, Phys. Rev. A 78, 022901 (2008).

[20] A. Schüller, H. Winter, M. S. Gravielle, J. M. Pruneda, and J. E. Miraglia, Phys. Rev. A 80, 062903 (2009).

[21] W. H. Miller, J. Phys. Chem. A 105, 2942 (2001).

[22] R. Guantes, A. S. Sanz, J. Margalef-Roig, and S. Miret-Artés, Surf. Sci. Rep. 53, 199 (2004).

[23] D. P. Dewangan and J. Eichler, Phys. Rep. 247, 59 (1994).

[24] M. Born and E. Wolf, Principles of Optics (Pergamon Press, Oxford, 1986), Chap. 10.

[25] C. Cohen-Tannoudji, B. Diu, and F. Laloë, Quantum Mechanics (Willey-VCH, Paris, 2011).

[26] A. Tonomura, Progress in Optics 23 (North-Holland, Amsterdam, 1986), p. 183.

[27] C. Keller, J. Schmiedmayer, and A. Zeilinger, Opt. Commun. 179, 129 (2000).

[28] I. Fabre, F. Navarrete, L. Sarkadi, and R. O. Barrachina, J. Phys.: Conf. Ser. 635, 042003 (2015).

[29] J. E. Miraglia and M. S. Gravielle (unpublished).

[30] F. W. Meyer, L. Folkerts, and S. Schippers, Nucl. Instrum. Methods Phys. Res., Sect. B 100, 366 (1995).

[31] A. Schüller and H. Winter, Phys. Rev. Lett. 100, 097602 (2008). 